\title{
Features and Design Intent in Engineering Sketches
}

\author{
Raquel Plumed $^{1}$, Peter Varley ${ }^{2}$, Pedro Company ${ }^{2}$ \\ ${ }^{1}$ Department of Mechanical Engineering and Construction \\ plumed@emc.uji.es \\ ${ }^{2}$ Institute of New Imaging Technologies \\ varley@emc.uji.es, pcompany@emc.uji.es \\ Universitat Jaume I, Castellón de la Plana, Spain
}

\begin{abstract}
We investigate the problem of determining design intent from engineering sketches: what did the designer have in mind when sketching a component? Specifically, we consider the unidirectional reverse mapping from form features, as determined from an input sketch, to design features, representing the design intent present in the designer's mind. We introduce a list of common engineering form features. For each, we list which geometrical cues may be helpful in identifying these features in design sketches, and we list the design features which such form features commonly imply. We show that a reductionist approach which decomposes a diagram into form features can be used to deduce the design intent of the object portrayed in a drawing. We supply experimental results in support of this idea.
\end{abstract}

\section{Introduction}

We aim to provide tools for design engineers who are already familiar with (and make good use of) the various features of stateof-the-art CAD packages, but who want the additional freedom offered by sketching when in the creative phase of designing a new product. Hence, we are interested in the interpretation of sketches of engineering components, both singly and in assemblies. In particular, we are interested in the problem of design intent.

When we define design intent as the concept which the designer had in mind when sketching the component (and not, for example, merely as a synonym for 3D parametric design, the ability to adjust objects and features by varying a few parameters), we see that perceiving design intent in sketches is not a simple problem. An engineer looking at a sketch sees: a) Geometry (which constraints are important?), b) Manufacturing features (how can the part be manufactured?) and c) Purpose (what does it do?). Design intent includes all of these, and they interact with one another in ways which can be subtle. In this paper, we focus on purpose or function: what does the object do?

Engineering sketches are a curious kind of a diagram, as they convey 3D shape information on two distinct levels: pictorial, where the 2D lines of the drawing portray the 3D object, and by means of engineering conventions such as non-pictorial lines and annotations (figure 18b). Anyone can interpret pictorial representations (although engineering training certainly helps in visualising the resulting 3D solids), and converting pictorial sketches into graphs and applying approaches based on graph processing has been a popular strategy for convert sketches into 3D geometrical models, i.e. graphics recognition [19] plus machine interpretation of line drawings $[17,7]$. But extracting all of the information implicit in such diagrams also requires both understanding engineering sketching conventions and using this understanding to modify the visualised 3D solid, and in practice only those with training in engineering or design are able to make full use of this extra information.

Our focus here is on the engineering training which aids designers in seeing the shapes and functions of 3D objects when they look at pictorial drawings. Designers use sketches as diagrammatic representations to communicate with one another and other designers can deduce meaning from such sketches, and this ability is a function of engineering training. This suggests that designers have a common knowledge base, a set of features which they can identify in drawings. Can we automate this interpretation process? Can we teach a computer to identify features?

We observe that designers perceive design intent embedded in sketches by recognising familiar features. Features, in turn, are perceived from combinations of hints or cues. In this paper, we argue that this paradigm corresponds to the way engineers habitually interpret drawings, and that this procedure may be replicated algorithmically.

To simplify the problem, we currently assume a single axonometric sketch which shows a general view of the object: no small change of viewpoint changes the topology. In this paper, we assume that there are no cut or exploded views (we hope to be able to relax this assumption in later work). The object is a Euclidean solid: it is essentially polyhedral; simple analytical curved surfaces such as axis-aligned cylinders are permitted; freeform curves are excluded. Formally, a Euclidean solid is one whose projections onto axis-aligned planes can be constructed using Euclidean tools: straight edges and conic arcs.

In Section 2, we discuss three relevant questions: why do designers use sketches? which kinds of feature do they perceive in sketches? how do they see features when they look at sketches? In Section 3, we introduce a list of geometric features, giving for each (a) the geometric cues which enable us to identify the feature in sketches and (b) the design intent which the presence of such a feature commonly implies. Section 4 gives examples which illustrate how consideration of form features can help determine the design intent and provides experimental verification that this gives similar results to human interpretation of design drawings. Section 5 presents brief conclusions and suggestions for future work.

We do not discuss vectorisation, the conversion of a freehand sketch into a graph-like line drawing. For the current states of the art of the various variants of vectorisation, see [14] for raster image input, [2] and [3] for scribbled sketches input and [24] for 


\section{Discussion: Sketches and Features}

\subsection{Role of sketches in engineering design}

Sketches assist product designers during the creative stages of design and help them to develop inventions. As Booker [4] writes, "The pencil has been called the most potent instrument in the world, for it gives most of man's thought and aspirations their first visible form”.

Ferguson [9] adds the observation that when two designers meet never just sit down and talk. They draw sketches for one another. They even take the pencil from one another as they talk and draw together on the same sketch. The sketch forms the basis of the discussion, as it provides all parties in the discussion with a common representation of the idea being discussed.

The common factor here is the use of the sketch as a guide, either for the designer's own thoughts or for communicating them to colleagues. We discuss this further in [5].

Thus, our audience is those design engineers who want:

- The freedom of pencil and paper to express different views of the object (orthographic, detailed, pictorial); scaffolding; highlighting; hidden lines; axes; hatching; overtracing (autocorrection, thinking, decoration) and annotating.

- The ability to add annotations: dimensions; cut views with hatching; icons; symbols.

- The power of pencil and paper to use this freedom and flexibility to communicate design intent.

The final point is the one we deal with in this paper. Frequently, it has tacitly (and wrongly!) been assumed that function is no longer of interest once the design has left the designer's mind and been expressed as geometry. We disagree strongly. Capturing only the geometry implied by sketches leaves us with only a low level description of the object. In contrast, the high level description which we call design intent is complex and useful information, and both the design intent and the sketches in which it is embedded should be preserved.

Automatic extraction of such complex information embedded in sketches is difficult. We advocate a reductionist approach: we try to infer design intent from features within the sketch. This can be contrasted with Yaner and Goel's holistic approach [23], which attempts to deduce design intent by matching the sketch with a template. Clearly, given a finite set of templates for which the design intent is known, the holistic approach can find which template best matches the drawing, and this gives a good idea of the design intent of the new drawing. Equally clearly, the holistic approach cannot be used for novel designs, for which no template is available.

For example, a reductionist approach to deducing the design intent of Fig. 1 would first note three features: a large flat base; a vertical cylindrical boss; and a vertical cylindrical hole or pocket, coaxial with the boss. We might then deduce that the cylindrical hole or pocket holds something in place, and from the need for a large base to hold the part firm, we might also deduce that whatever fits in the hole or pocket is expected to move, possibly by rotating about the axis.

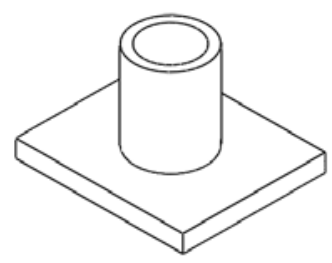

Fig. 1. Combination of features

Additionally, designers need to be free to choose the order in which things are created. Typically, things which express design intent come first. Decorative detail can be left until last, and some secondary features, not related to the object function, can be ignored entirely at this stage if they can be added easily to the CAD model.

\subsection{Kinds of Feature}

Vandenbrande and Requicha [20], arguing against the then (1993) current paradigm of designing in terms of manufacturing features, distinguish machining features, which are produced by a specific machining process and thus necessarily subtractive, from design features, sketched configurations which, in the designer's mind, have a particular purpose related to the function of the object. They point out, in particular, that manufacturing technologies may change several times during the object's lifetime, but it retains the same function throughout. On this basis, objects should be designed in terms of their function, not in terms of manufacturing technology.

To this, Han $[10,11]$ adds form features, geometric descriptions of shape with no implied relation to function or manufacturing 
method. He recommends using form features during the design process and subsequently converting the finished design to manufacturing features, and he documents methods for converting form features present in CAD models into machining features for particular manufacturing technologies. Others have identified many other categories of feature: analysis, material, tolerance ... These are unrelated to the problem of interpreting pictorial representations, and we can safely ignore them.

Thus, there are three important types of feature: design features in the designer's mind turn into form features in the sketch and $\mathrm{CAD}$ model, and these are later interpreted as machining features for process planning. The mappings between any two of these sets are not one-to-one.

From [20] onwards, there has been a good deal of research in conversion of form features to machining features. Feature-based design has also developed in the last twenty years, moving from feature recognition for manufacturing purposes and developing into a broader method for interactively designing by features [16], and is now a well established research area. But very little research in automatically deducing design features has been done.

In Section 3, we present a catalogue of common form features and attempt to relate them to design intent. We take the online catalogue of CustomPartNet [8] as our starting point, as it is based solely on form features, whereas feature libraries associated with CAD/CAM typically have a close association with machining process, as do the catalogues used in reverse engineering such as Thompson et al. [18].

It will be noted that we have excluded from our catalogue a feature very popular in CAD: extrusion. Extrusion is both a quite convenient CAD modelling operation and a manufacturing feature, but extrusion as such does not map to any particular design intent. However, some form features which can be modelled and manufactured by extrusion have been included when these are linked to a recognisable function-for example, rails are used for guidance during relative displacement between two parts.

More difficult is the question: should the catalogue include fillets and rounds? We are inclined to think not. The functions of fillets and rounds are to remove sharp edges and sharp transitions (which could hurt people, are difficult to machine, concentrate stresses, etc), and this is a secondary function, not the primary function which we seek when determining design intent. Also, fillets and rounds can be added easily in CAD packages, and thus decoupled from input sketches [6].

Finally, we do not consider composite features, such as the mounting boss in Figure 2, where six form features (a drilled cylindrical boss reinforced by four triangular ribs) combine to perform a single function (fastening one corner of this part to another part with a screw). Neither do we consider distributed features, such as the three legs of the tripod in Figure 2, which are a single functional feature (stand the object stably on an uneven surface) even though they are topologically separate. These are too complex for an initial catalogue. We hope we will be better able to deal with them once we can identify their elements (e.g. for the mounting boss, a long cylindrical boss surrounded by regularly placed ribs).
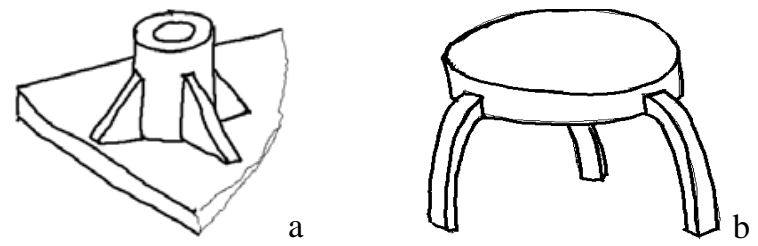

Fig. 2. Mounting boss (a) and tripod (b)

\subsection{Finding Features in Sketches}

Recognition of features from 3D input is a well-established (albeit still open) problem [1], whereas recognition of features from 2D input has barely been considered. According to Han et al. [13], there are three promising classes of feature recognition approach: graph-based approaches, volumetric decomposition approaches, and hint-based approaches. The last of these is similar to the perceptual approach of Sketch-Based Modelling (SBM): Han's 3D traces or hints are little different from SBM's 2D regularities, clues or cues - they suggest the possible existence of a feature. Clearly, algorithms developed to analyse 3D input cannot be applied directly to 2D input, but they may provide a useful starting-point.

Before we can apply current feature recognition approaches to 2D sketches, we must infer depth information from cues within the sketch. See Varley [21] for a framework for creating 2⿺辶⿳亠丷厂 D and 3D models from natural line drawings of polyhedra.

Alternatively, we can search for features directly from sketches.

Where there are established algorithms for detecting particular form features, we include citations to them in Section 3 . In most cases, these algorithms were designed for 3D input, and a non-trivial conversion would be required to use them with 2D inputs.

There are several subtleties which must be taken into account when developing algorithms for identifying and distinguishing between form features.

Topology gives the most important cues for finding form features, as form features are generally defined by their topology. This is why, for instance, Fig. 16a is readily perceived as a rib, but the rectangular element in the middle of Fig. 17a is not-its rectangular shape prevents structural engineers from seeing it as a rib, since they know that there is no structural advantage in extending the optimal triangular shape to a rectangle. It may make sense as a division, if we know which other parts are to be assembled with it, and we can detect some advantage in preventing or limiting relative movements between them, but perceiving it as a division requires global knowledge about the topology of the part, and inferences about how it assembles with other related parts. 
Geometrical cues are generally less important, but cannot be ignored entirely. For example, aspect ratio is a necessary cue in the case of the "fat rib" in Fig. 16c.

\section{List of Form Features}

This section presents a list of common form features. For each, we outline how the feature may be identified from its typical geometry, and how it may map to one or more design features.

Where reliable algorithms for detecting the feature exist, we include a citation. The most useful algorithms are those which return probabilities or merit figures: instead of just saying whether or not a particular cue matches an idealised template, the algorithm determines how well a cue in an imperfect drawing (derived from a hand-drawn sketch) matches the template.

As an alternative to developing specific algorithms for each form feature, we could use a template-matching approach such as that of Yaner and Goel [24] to find features. However, this would require that, for each form feature, we have templates which illustrate that feature in all of its common aspects.

\subsection{Slot}

Geometry: A slot is a channel opened on a usually flat surface by removing material. The geometrical information available in the drawing depends on the orientation (compare fig. 3a vs. fig. 3b). The channel is long and narrow and typically extends the full width of the surface, but slots can be completely contained within all sides of the part, or extend to one side. The extruded subtractive profile need not be rectangular: dovetail slots, as in Fig. 3c, and more complex shapes, as in fig. 3d, are also possible, and the depth of the slot can extend through the part.
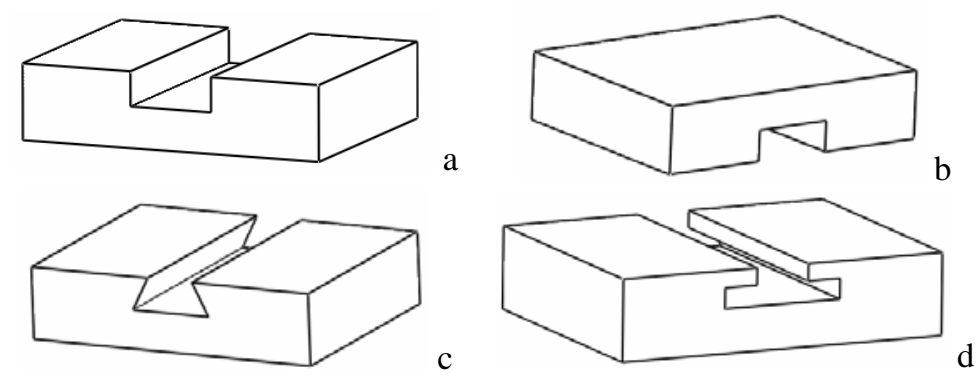

Fig. 3. Slots

Slots typically follow a straight path and are passing through, but can also be blind (fig. 4a), and follow any simple or complex path (fig.4b). They may also appear in groups (fig. 4c) and have varying profiles (fig. 4d).

As slots are subtractive, slots translate directly into machining features.

The geometric cues which should be considered when assessing whether a (wireframe) drawing depicts a slot are:

- Are the bottom face and the side faces of the potential slot quadrilateral? (N.B. since a quadrilateral face in 3D is depicted as a parallelogram in a 2D drawing, the algorithm should search for closed circuits of four edges with opposed pairs of edges parallel.)

- Do opposed pairs of side faces have the same shape? Are they symmetric from a symmetry plane located in the longitudinal axis of the slot? (N.B. 3D bilateral symmetry can be detected in the drawings as skewed mirror symmetry [15].)

- Are there two or more coplanar faces? Are there two or more collinear edges? This cue suggests that a larger faces has been interrupted by a feature such as a slot, as in the examples in fig 3.

- Do the end cross-sections (open slot, fig 3) or end caps (closed slot, fig 4) have the same shape? Does the symmetry plane of the cross-section match up with the symmetry plane located in the longitudinal axis of the slot? Typically, the machining procedure of milling produces slots with constant cross-section along the longitudinal axis.

- Is any long edge of the bottom face on the drawing boundary? If so, the feature cannot be a slot. (However, it can still be a partial slot, missing a side face, as in fig 4c.)

- Are there bosses within the bottom face? If so, the main feature cannot be a slot. (N.B. holes within the bottom face would not obstruct the complementary part, so are permitted.)

Design Intent: Slots are aimed at obtaining a fix or sliding fixture with a complementary part (i.e. things are tied to it or slide along it). Slots are a strong clue that the object is part of an assembly, and provide topological and geometric information about how the assembly is to be assembled. The partner piece is likely to be a rail, as in Section 3.2. 

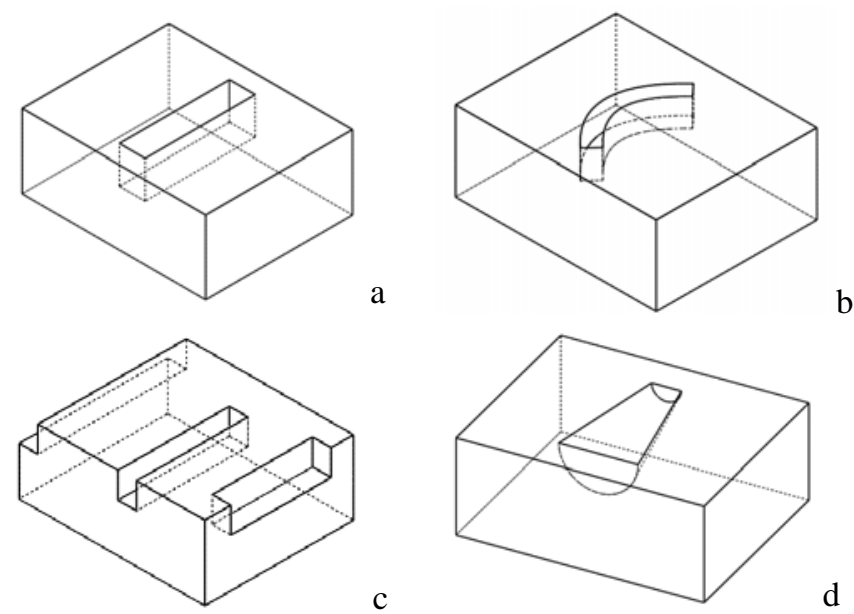

Fig. 4. Different paths (a and b), multiple (c) and contained (d) slots

Detection: There are well-known algorithms for detecting slots. For example, Han and Requicha [12] identify a slot cue from nominal geometry when a pair of planar, parallel, opposing faces is found. Varley [21] looks for underslots and valleys by trying to match the region around each T-junction in the drawing with the templates shown in Figure 5 (however, Varley [21] is concerned with geometric reconstruction, not interpretation, so, for example, Fig $3 \mathrm{~b}$ is identified as a slot because this aids reconstruction, but Fig 3a is not identified as a slot because reconstruction is straightforward anyway).
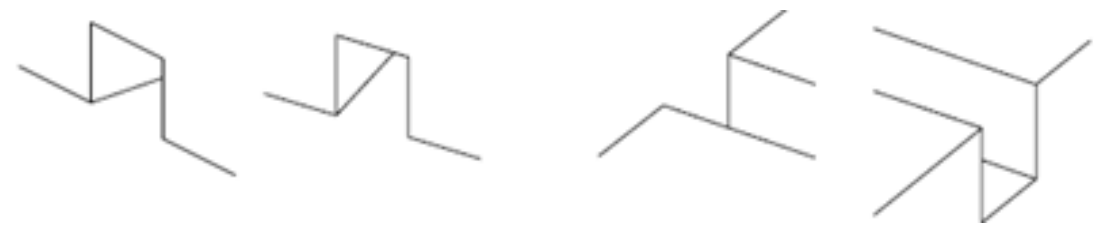

Fig. 5. Slot templates

\subsection{Rail}

Geometry: Rails are longitudinal protrusions (some sources name them longitudinal ribs). Rails are long and narrow and have a constant profile, usually rectangular (fig. 6a) but sometimes not (fig. 6b), usually following straight paths but sometimes following complex paths (fig. 6c) and usually located on top of flat surfaces but sometimes not (fig. 6d).
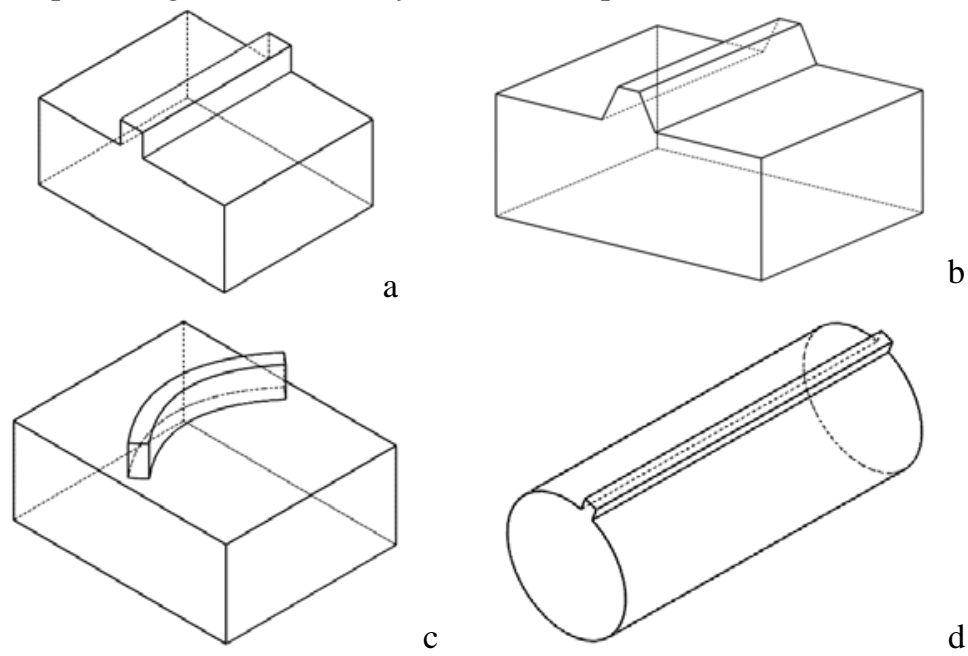

Fig. 6. Rails

The geometric cues which should be considered when assessing whether a (wireframe) drawing depicts a rail are:

- Are the upper face and the side faces of the potential rail quadrilateral? (Note this is the same test as was used for slots.)

- Do opposed pairs of side faces have the same shape? Are they symmetric about a symmetry plane located along the longitudinal axis of the rail? (Again, note this is the same test as was used for slots.) 
- Are there two or more coplanar faces? Are there two or more collinear edges? This cue suggests that a larger faces has been interrupted by a feature such as a rail, as in the examples in figs $6 \mathrm{a}$ and $6 \mathrm{~b}$.

- Do the end cross-sections (open rail, figs 6a and 6b) or end caps (closed rail, fig 6c) have the same shape? Does the symmetry plane of the cross-section match up with the symmetry plane located in the longitudinal axis of the slot?

- Is any edge of the upper face on the drawing boundary? As rails are protrusions, any edge of the upper face can be a boundary edge. This test can be used to distinguish rails from slots.

- Are there bosses within the upper face? If so, the main feature cannot be a rail. (N.B. holes within the upper face would not obstruct the complementary part, so although rare they are permitted.)

Design Intent: Rails are a strong clue that the object is part of an assembly, and provide topological and geometric information about how the assembly is to be assembled. A single rail usually conveys the design intent of sliding joint between two parts (fig. 7a). Uniformly arranged sets of rails usually convey fixed joints, as in fig. 7b. The partner piece is likely to be a slot, as in Section 3.1.
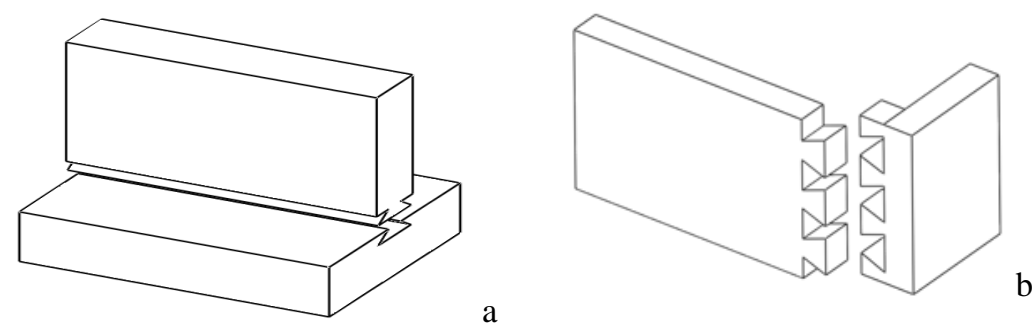

Fig. 7. Assemblies with sliding dovetail join (a) and half blind dovetail joint (b)

Detection: As far as we know, no algorithms for detecting rails have been reported.

\subsection{Pocket}

Geometry: A pocket is an extruded subtractive volume which does not meet the criteria to be considered a hole or a slot: not as deep as a hole (figs. 8a and 8b), and not as long as a slot (figs. 8c and 8d). If a feature has a uniform profile along its depth but has a low aspect ratio it is considered a pocket, not a hole (fig. 8a). A feature with a high aspect ratio but a non-uniform profile is also a pocket, not a hole (fig. 8b). A feature with a uniform extruded subtractive profile along a short length is considered a pocket, not a slot (fig. 8c). Also, a long feature with a non-uniform profile along its length is considered a pocket, not a slot (fig. 8d).

The geometric cues which should be considered when assessing whether a (wireframe) drawing depicts a pocket are:

- Does the candidate pocket meet the topological criteria for a slot? (See Section 3.1.)

- Does the bottom face have a larger area than the side faces? This is the geometric criterion which distinguishes pockets from slots.

- Are there smaller bosses embedded within the bottom face? If so, the main feature cannot be a slot but can still be a pocket.
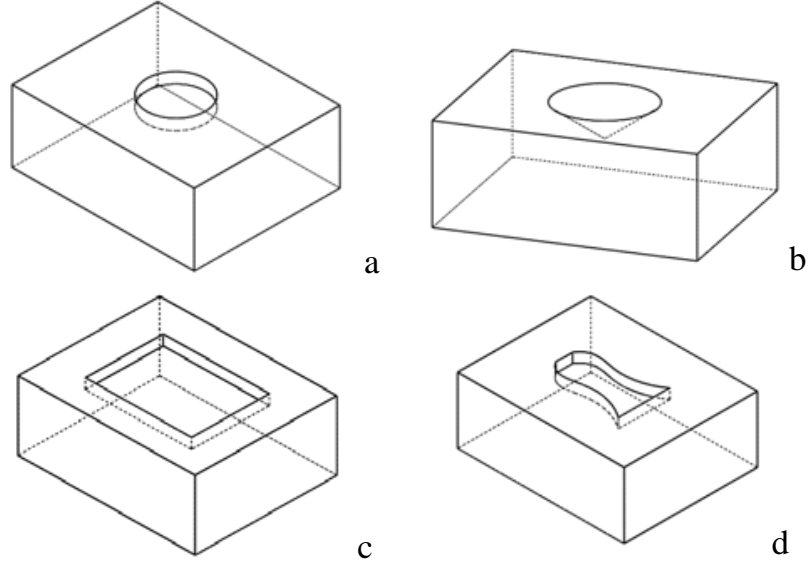

Fig. 8. Pockets

Pockets may have complex regular or irregular borders (fig. 9a and 9b). A pocket need not necessarily have a flat base (fig. 9c). Pockets which combine many of the elements described above (irregular and non-uniform profile, contoured base) can take on a highly complex form, and are better classified as sunk reliefs (fig. 9d) - N.B. since reliefs are decorative, not functional, we do not consider them here. 

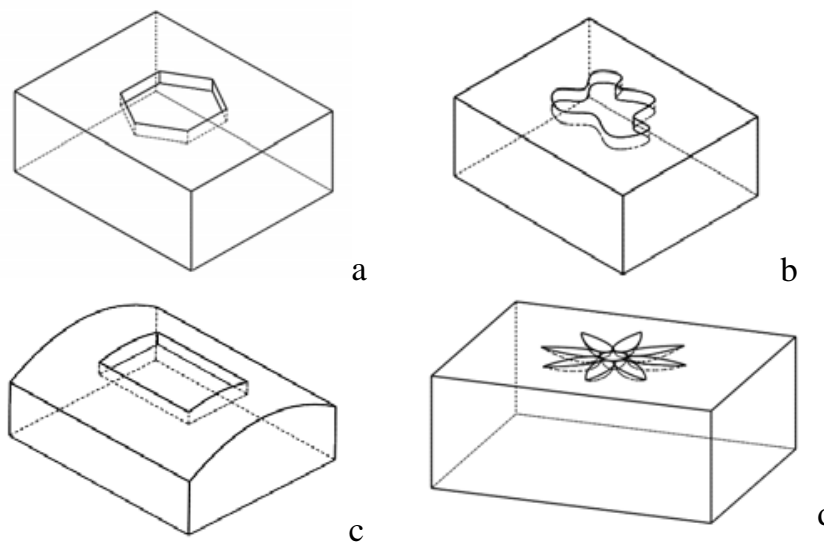

Fig. 9. Complex pockets

Most pockets are contained within a single face of the part, but pockets can also extend to two or more faces. Multiple pockets can occur in the same face.

Design Intent: Pockets are a strong clue that the object is part of an assembly, and provide topological and geometric information about how the assembly is to be assembled. The partner piece is likely to be a step, as in Section 3.4, or a boss, as in Section 3.5.

Alternatively, pockets within the top face of an object could be intended as reservoirs to hold liquids, or as trays to catch falling objects.

Another alternative for pockets on the top face of an object is that they are intended as moulds as part of a manufacturing process: a liquid is poured into the mould and sets to create the desired object.

Detection: As pockets are subtractive, they translate directly into machining features. There are well-known algorithms for detecting them. See Shahin [16] for an overview which describes various form-feature recognition methods and explains the fundamentals of each method and the main differences between them. Also, Company et al. [5] present an inflation engine which automatically reconstructs polyhedral sketches. The process filters out sketch imperfections and then recognises skeleton shapes using an approach which combines geometry recognition (line, arc, ...) and recognition of symbols which represent constraints (parallel, perpendicular, ...). It works reasonably well with polyhedral skeletons of many shapes, including those with pockets.

\subsection{Step}

Geometry: A step is an extruded additive volume with a low aspect ratio (the feature is wider than it is tall), and a regular (fig. 10a) or irregular (fig. 10b) contour. A step can have a variety of extruded additive profiles (fig. 10c) and can be uniform or nonuniform along any direction (fig. 10d). Also, a step can be a flat surface or a contoured shape. When a step combines many of the elements described above, such as an irregular and non-uniform cross section and a contoured top, it can take on a highly complex form and is better classified as a bas-relief.

The geometric cues which should be considered when assessing whether a (wireframe) drawing depicts a step are:

- Does the candidate step meet the topological criteria for a rail? (See Section 3.2.)

- Does the bottom face have a larger area than the side faces? This is a geometric criterion which distinguishes steps from rails.

- Are there smaller bosses embedded within the upper face? If so, the main feature cannot be a rail but can still be a step.
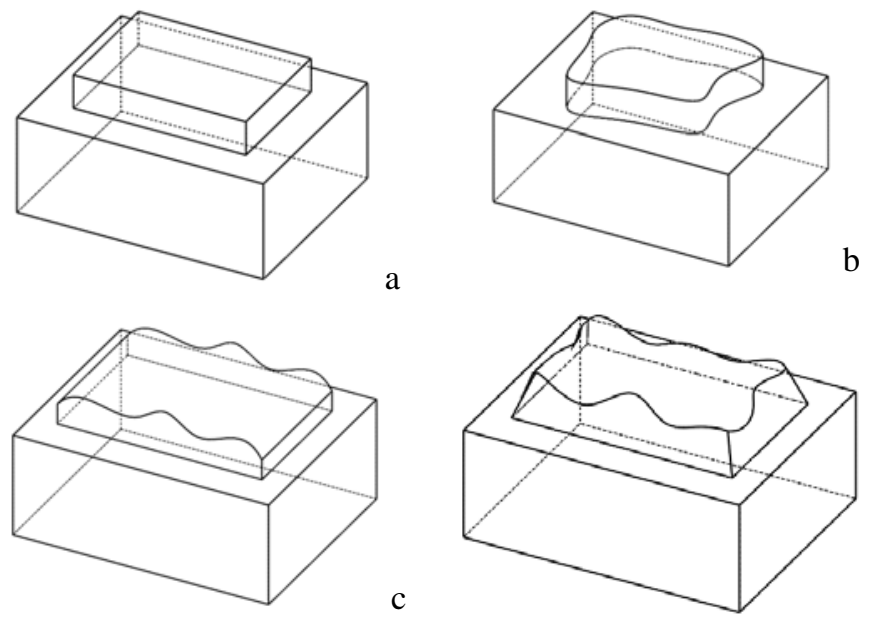
Design Intent: Steps are a strong clue that the object is part of an assembly, and provide topological and geometric information about how the assembly is to be assembled. The partner piece is likely to be a through pocket, as in Section 3.3.

A profile island is a special kind of step (removing rounds and fillets, as in [6], converts many islands into normal steps). It is defined only in the context of a pocket and specifies a volume to be skipped when the pocket is milled. It provides a flat surface of support around one or more holes (fig. 11).

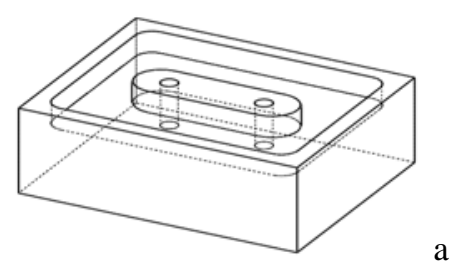

a

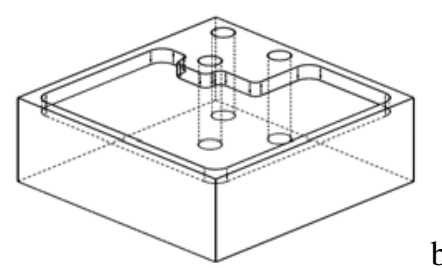

b

Fig. 11. Central island (a) and corner island (b)

Detection: As far as we know, no algorithms for detecting steps have been reported.

\subsection{Boss}

Geometry: A boss is an extruded additive volume with a high aspect ratio (fig. 12a). In a sense, it is the inverse of a hole, but does not necessarily require a uniform profile (fig. 12d). Most bosses have a circular profile (fig. 12b). However, a boss can have a polygonal, irregular (fig. 12c), or non-uniform extruded additive profile. Multiple bosses protruding from the same face are possible.

The geometric cues which should be considered when assessing whether a (wireframe) drawing depicts a boss are:

- Does the candidate step meet the topological criteria for a rail? (See Section 3.2.)

- Does the bottom face have a smaller area than any of the side faces? This is a geometric criterion which distinguishes bosses from rails.
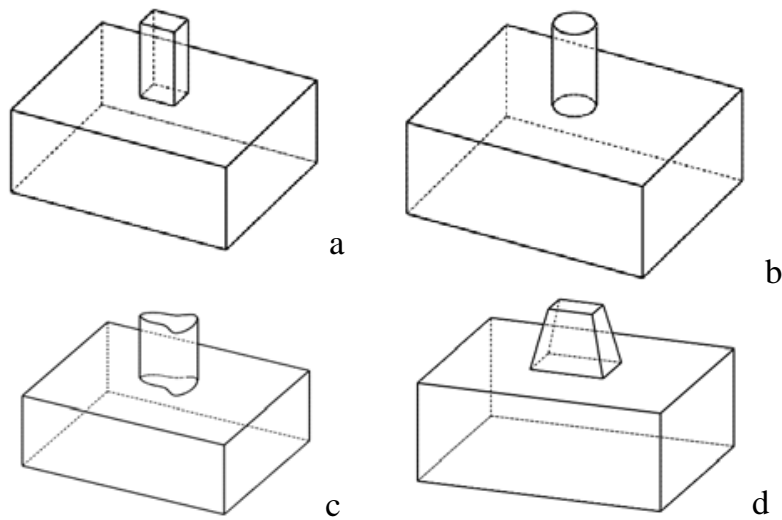

Fig. 12. Bosses

Design Intent: The boss fits in a pocket. Bosses are a strong clue that the object is part of an assembly, and provide topological and geometric information about how the assembly is to be assembled. The partner piece is likely to be a pocket, as in Section 3.3. Detection: Varley [21] identifies topologically-simple polyhedral bosses such as Fig. 12a and Fig. 12d by analysing junction labels of the inner and outer loops of a multi-loop face.

\subsection{Hole}

Geometry: A hole is a subtractive volume with a uniform profile along its depth and a high aspect ratio (the depression is deeper than it is wide). The extruded subtractive profile need not be a rectangle. Most holes have a circular profile and form a cylindrical hole (fig. 13a). However, a hole can have a polygonal or even an irregular profile (fig.13b). Multiple holes, as in fig. 13c, are also possible. The distinction between through and blind holes (fig. 13d) is important geometrically as each through hole increases the genus of the object by one (blind holes do not modify the genus of the base shape).

The geometric cues which should be considered when assessing whether a (wireframe) drawing depicts a hole are:

- Does the candidate hole meet the topological criteria for a slot? (See Section 3.1.) 
- Does the bottom face have a smaller area than any of the side faces? This is the geometric criterion which distinguishes holes from slots.

- Is the cross-section circular? While not all holes are circular, circular holes are particularly common. (N.B. as circles in 3D are projected to ellipses in axonometric views, algorithms should look for elliptical curves as cues to the presence of holes.)
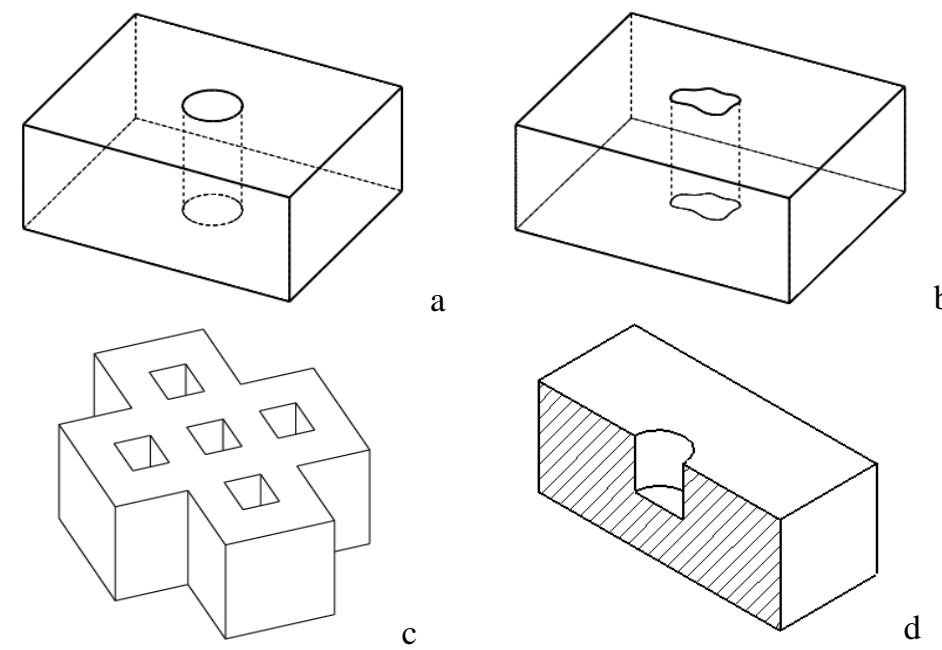

b

Fig. 13. Through holes and Blind holes

Design Intent: A single hole of a simple profile (rectangular or circular) is a clue that the object is part of an assembly, and provides topological and geometric information about how the assembly is to be assembled. In such cases, the partner piece is likely to be a boss, as in Section 3.5.

Holes of complex profiles, and multiple holes, could also be a clue that the object is part of an assembly, but a more common interpretation is that the object is a mould/cast for creating objects by extrusion as the material to be moulded/cast is pressed through the object.

Holes with a circular profile, whether single or multiple, could in principle be the base for screw holes. However, screw holes are a secondary feature which is easy to add using a CAD package, so designers will more often than not omit screw holes when sketching.

Detection: As holes are subtractive, they translate directly into machining features. There are well-known algorithms for detecting round holes: see Shahin [16] for a recent summary.

\subsection{Oblong Hole}

Geometry: Oblong holes are short rectangular slots finished with semicircular contours (fig. 14a). They are usually through holes. Topologically, oblong holes are indistinguishable from through holes (Section 3.6) - the differences are the shape and aspect ratio. Multiple oblong holes, as in fig. 14b, are also possible.

The geometric cues which should be considered when assessing whether a (wireframe) drawing depicts an oblong hole are:

- Does the candidate oblong hole meet the topological criteria for a slot? (See Section 3.1.)

- Is it a through hole? Both end caps must interrupt other faces.

- Does the cross-section match that of an oblong hole? The cross-section should be a parallelogram with semicircular arcs at the shorter opposed edges. (N.B. part-ellipses in the drawing are a cue to the presence of oblong holes.)
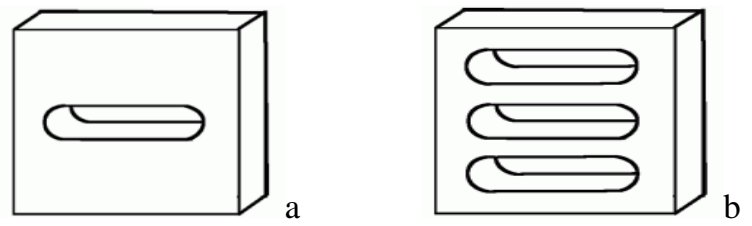

Fig. 14. Simple (a) and multiple (b) Oblong Holes

Design Intent: One oblong hole is usually there to allow for misalignment of screws or bolts. Two or more oblong holes are usually for ventilation, to allow hot air to escape, or maybe just to reduce the weight. In either case, these are secondary features, not related to the primary function of the object, which is most likely to be covering a vent or an opening.

Detection: As holes are subtractive, they translate directly into machining features. There are well-known algorithms for detecting oblong holes [16]. 


\subsection{Spotface/Counterbore/Countersink}

Geometry: Spotfaces (fig.15a) and counterbores (fig. 15b) are cylindrical recesses, typically machined around a hole. A spotface is a shallow counterbore-the difference is the aspect ratio. Countersinks have conical-shaped recesses and may be topologically identical or may omit the lower ring (fig. 15c). These features are typically machined into cast parts, but other locations are possible-they can, for example, be on the top of bosses.

These features could be considered as special types of holes, as they are effectively holes with different sections along their axes of revolution.

The best way to identify these features algorithmically would be to combine the algorithms for identifying pockets and holes, and find those pockets and holes which have collinear axes of revolution.

Design intent: The purpose of these features is to house a fastener (bolt head, nut, screw) so that these sit flush with the part surface. Spotfaces and counterbores offer flat faces against which to seat a fastener. In addition, spotfaces provide flat surfaces perpendicular to the bolt axis to ensure uniform loading on the bolt face. Countersinks make it easy to line the fastener up with the hole by guiding it into place. The diameter must be larger than that of the head of the bolt (across corners if polygonal).
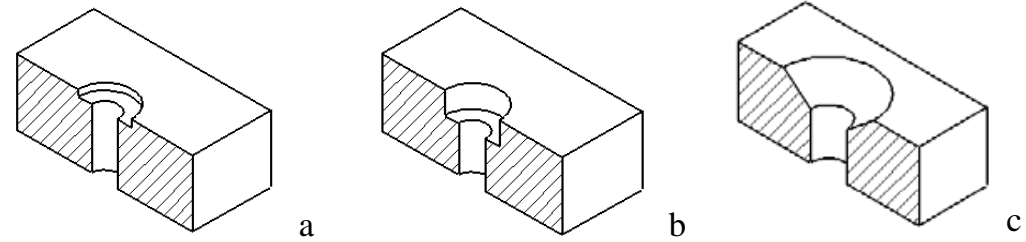

Fig. 15. Spotface (a), counterbore (b) and countersink (c)

Detection: As spotfaces, counterbores and countersinks are subtractive, they translate directly into machining features. There are well-known algorithms for detecting them [16].

\subsection{Rib}

Geometry: A rib is a triangularly shaped protrusion which connects two surfaces. A rib is typically used between a wall of a part and the flat surface from which it protrudes. Local geometry alone does not always enable us to distinguish ribs from dividers (section 3.9)—we must consider the geometry of the whole object. Multiple ribs, as in Fig. 16b, are also common.

Aspect ratio can be of importance in identifying ribs. Figures 16a and 16c are topologically identical: the only difference is the thickness of the central feature. The feature in Figure 16a would generally be interpreted as a rib, but that in Figure 16c would not: the common function of ribs is to increase strength without adding too much weight, and the "fat rib" in Figure 16c is too heavy. (This leaves open the question: how should we interpret Figure 16c? Perhaps it should be seen as a wedge block with two lateral pockets).

Since the most distinctive geometrical aspect of ribs is that they are thin walls, the initial stage when detecting ribs should be searching for slender parallelograms depicting thin border faces: quadrilateral faces with two quite short opposed edges and two longer edges. When one is found, the sides of the candidate rib, the two faces connected to the longer edges of the border face, should be equal.

In addition, as ribs interrupt the faces in which they are embedded, edges connected to the vertices of the ribs' side faces but which do not belong to the rib should be collinear.

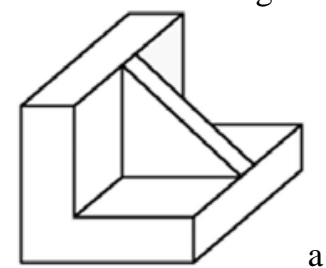

$\mathrm{b}$

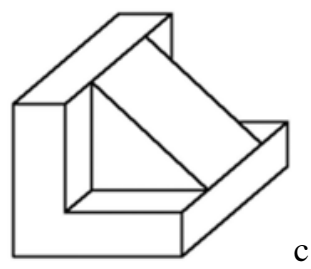

Fig. 16. Single rib (a), multiple ribs (b) and not-a-rib (c)

Design Intent: Ribs reinforce the structure without adding too much to weight or cost. Note that some designers include ribs in their sketches while others do not, as ribs are a secondary feature, not related to the primary function of the object. Ribs are also used to support other features, such as bosses (fig. 16b).

Detection: As far as we know, no algorithms for detecting ribs have been reported.

\subsection{Divider}

Geometry: A divider is a thin wall which partitions the region around it into two halves (fig. 17a). Note that local geometry alone 
does not always enable us to distinguish ribs from dividers-we must consider the geometry of the whole object.

Multiple dividers, as in fig. 17b, are also possible.

Algorithmically, we can distinguish probable ribs from probable dividers. Dividers are likely to have more than one border face, and their lateral faces are likely to be quadrilateral, so the presence of these cues reduces the likelihood of ribs and increases that of dividers.

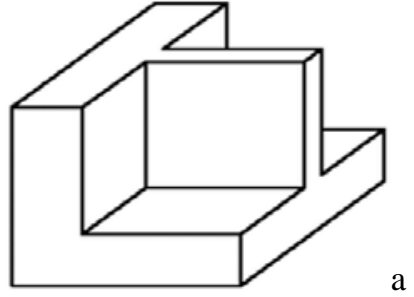

a

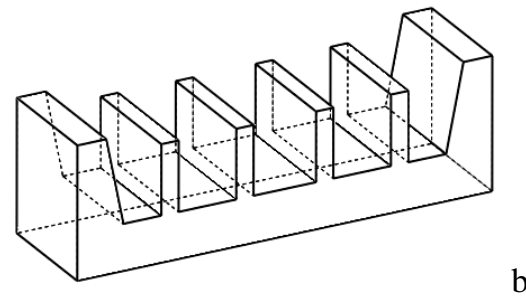

$\mathrm{b}$

Fig. 17. Single divider (a) and multiple dividers (b)

Design Intent: Dividers could be present simply to partition space into separate regions. They could also be present to limit movement with respect to another part.

Alternatively, multiple dividers may be cooling fins. If we can deduce that cooling is a global requirement, we can further deduce that the central element plays this role, but this requires knowledge about the global function or behaviour. However, we note that cooling fins are usually used in groups and arranged following regular patterns, and this may give us the required topological cues to detect them.

Detection: As far as we know, no algorithms for detecting dividers have been reported.

\subsection{Base Plate}

Geometry: There is no standard geometry for base plates - the important clues are (a) that there is a large planar surface on the underside of the object and (b) that nothing protrudes below this planar surface (fig. 18a).

A specialised example of base plate is a flange, defined as a flattened collar or rim around a cylindrical part to allow for attachment (fig. 18b).

The most useful cues to the presence of a base plate are that (a) the main face is outermost, not a feature embedded in any larger face, (b) the area of the main face is considerably larger than the area of any adjoining side face, and (c) that many of the edges of the main face or side faces are on the drawing boundary.

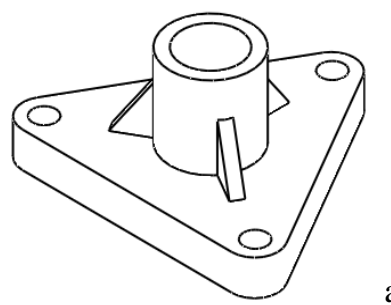

a

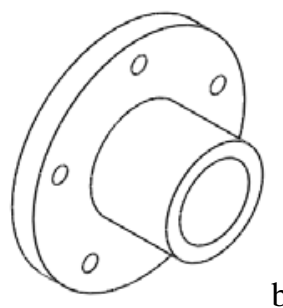

b

Fig. 18. Base plate (a) and flange (b)

Design Intent: A base plate allows the object to rest on a surface and allows for its attachment.

Detection: As far as we know, no algorithms for detecting base plates have been reported.

\section{Experiments}

Finally, we examine how design intent may be deduced by breaking down objects into features. We have shown how simple shapes such as the hollow boss in Figure 1 can be analysed in terms of its component form features. Can this process be applied to more complex objects?

More formally, we envisage a reductionist process in which: (i) all of the design features are identified (along with merit figures which record how well the candidate feature matches its ideal template); (ii) a combination table is constructed to record which candidate features are compatible and which are contradictory; and (iii) some form of statistical processing is used to determine which combination of candidate cues is the best interpretation of the drawing.

We carried out a first experiment in order to determine whether (a) this is possible and (b) engineers analyse drawings in this way. We supplied test subjects (most of them with an engineering background) with engineering drawings, and invited them to determine the features of the object depicted in each drawing, given their own engineering experience and common sense (fig.19). 
We used drawings of ten different objects (fig. 20), giving only five of them to each subject to prevent fatigue. We used two different illustrations of how to answer the test, to avoid illustrations which matched exactly with identical features in the parts. Each drawing was given on a separate sheet, giving subjects enough space to add marks and comments, and ensuring that they did not inadvertently compare different examples.

The subjects were mainly university teachers, including industrial engineers, mechanical engineers and industrial designers. We were looking for a population with technical training, as basic knowledge in engineering parts was required.

\section{FEATURES QUESTIONNAIRE}

We ask you to mark and identify, in the attached figures, those parts of the drawing that you believe they represent features, and describe, with a short annotation, its function in the object.

We understand by feature one geometrical configuration in the drawing which in the designer's mind has a particular purpose related with the function of the object (or part).

Given that the figures are not contextualized, and no additional information is available, some features may have ambiguous functions. In such cases, please, describe the function that you believe is most suitable according to your criterion.

The next illustration shows how to answer the test:

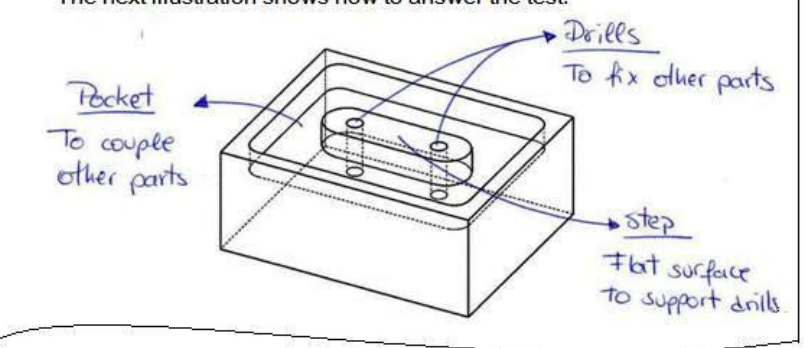

Fig. 19. Features questionnaire

We collected a minimum of 11 and a maximum of 14 different opinions for each of the ten drawings. In the central column of figure 20, we list, for each drawing, all the features perceived by the subjects, and in the right-hand column the degree of agreement in perceiving them.

\begin{tabular}{|c|c|c|}
\hline Object & $\begin{array}{c}\text { Agreement in } \\
\text { perception of } \\
\text { features }\end{array}$ \\
1 & $100 \%$ \\
2 & $100 \%$ \\
3 & $25 \%$ \\
4 & $58 \%$ \\
5 & $17 \%$
\end{tabular}




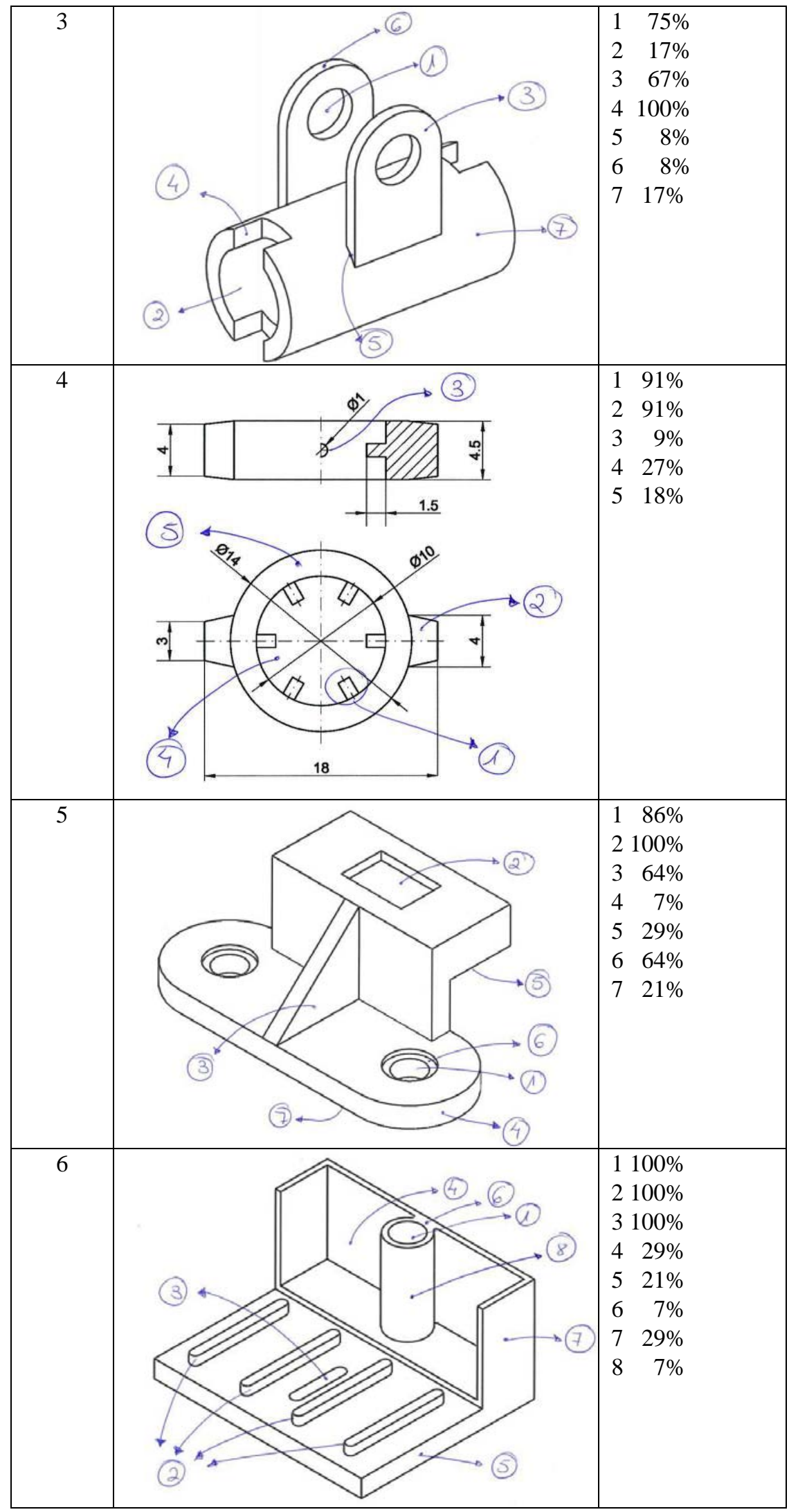




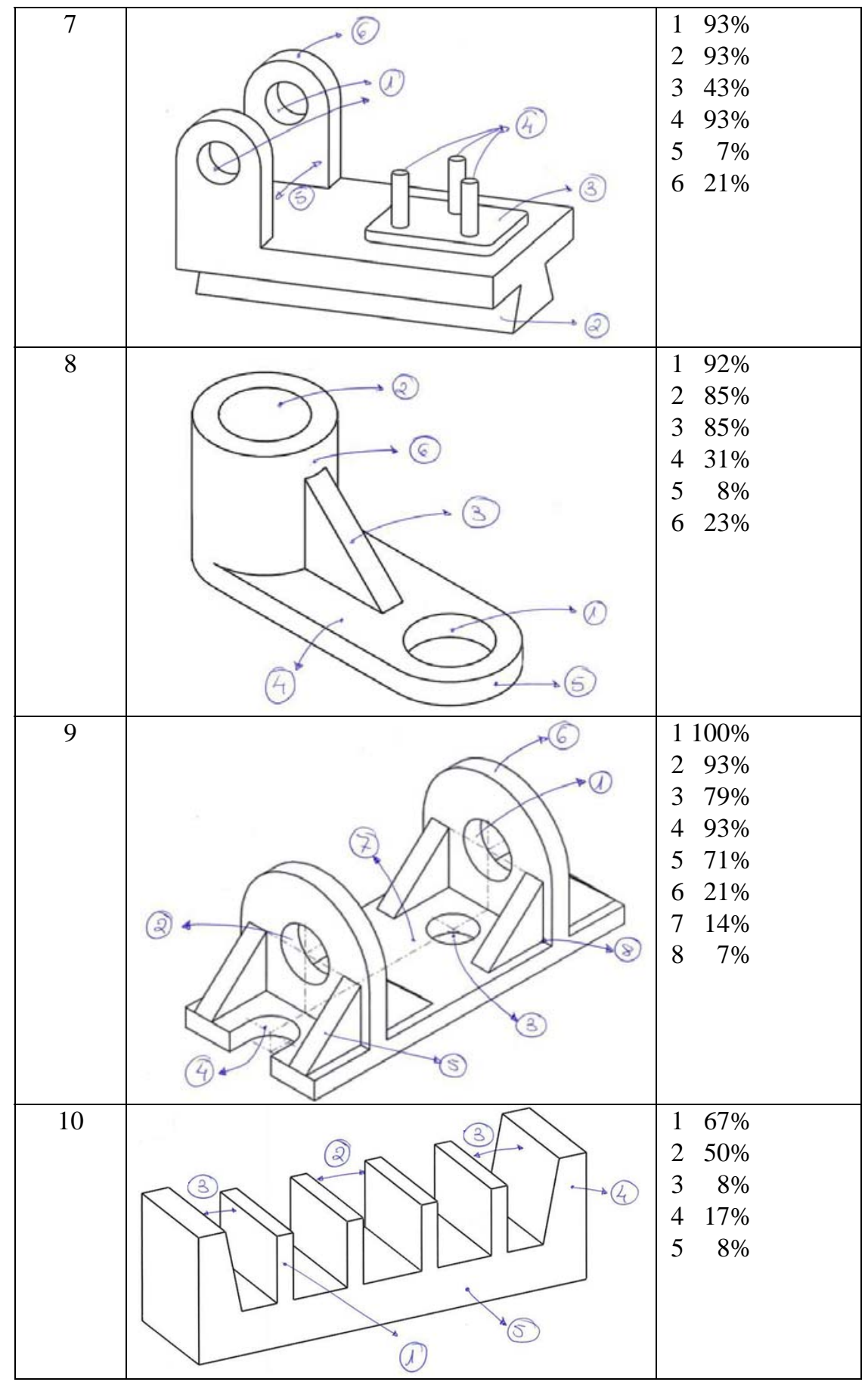

Fig. 20. Drawings, and features perceived by the interviewed subjects

Firstly, this experiment clearly validates the hypothesis that designers have a common knowledge base, a set of features which they can identify in drawings. Ten of the features depicted in the drawings were perceived by all of the subjects. At least one third of the features (22 out of 65) may be considered as "common", as they were perceived at least by $80 \%$ of the subjects. Only one third (17 out of 65) may be considered as "difficult" or "unusual" features, as they were perceived by under $20 \%$ of the subjects (again, the subjects agree about what they see, but this time they think the feature is not present).

Secondly, although the names given by different subjects to each feature sometimes varied (not least because different subjects spoke different languages!), there is reasonable evidence that their understanding of function was the same. For example, feature 2 in object 1 was labelled by 75\% of the subjects as a "chavetero" (keyway, in English). The remaining subjects explained the same functionality, although they gave a different name to the feature.

Our impression, resulting from informally talking with some of the subjects after they had completed the test, is that when multiple interpretations of a feature happen, they usually correspond to different ways to machine the feature (e.g. cylindrical hole vs. drill), rather than to different perception of design intent. It seems that, even two decades after Vandenbrande and Requicha, knowledge about machining features still influences the perception of design features to some extent.

Some of the subjects spontaneously wrote their perception of the function/design intent of the whole object. In order to get more extensive information on whether the drawings convey enough information about the design intent of the whole object, we performed a second experiment. In this, we explicitly asked the subjects to determine the design intent of the object, given the drawing and their own engineering experience and common sense. 
In the second experiment, our subjects were asked to describe the general function of three objects, outlining their reasons for their perceptions (fig 21). Each object was portrayed individually on a sheet where the subjects were able to answer and add their own comments. Neither additional information on the object nor information on its context was given. Again, the subjects were mainly professors from various departments of the same university, and included industrial engineers, mechanical engineers and designers; in addition, three students with postgraduate studies in engineering took part in the experiment. We were looking for a population with technical training, as basic knowledge in engineering parts was required.

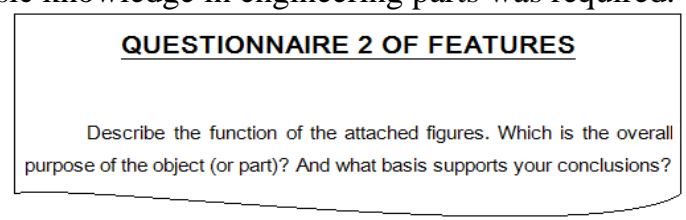

Fig. 21. Second features questionnaire

We collected a total of 16 opinions for each of the three examples. The results are discussed in following subsections. For each of our three examples, we give the drawing and its provenance, our own expectations of how it would be analysed, the results of the experiment, and an analysis of how well the results matched our expectations.

We highlight that some interviewed subjects gave multiple answers, proposing different ideas simultaneously. Hence, the number of subjects who support different functionalities does not always total $100 \%$.

\subsection{Example 1}

The first thing we notice in Fig. 22 is that it has a central axis which is concentric with four cylindrical extrusions. The object is clearly designed to rotate about this axis: it is a shaft. This particular object is in fact a propeller shaft.
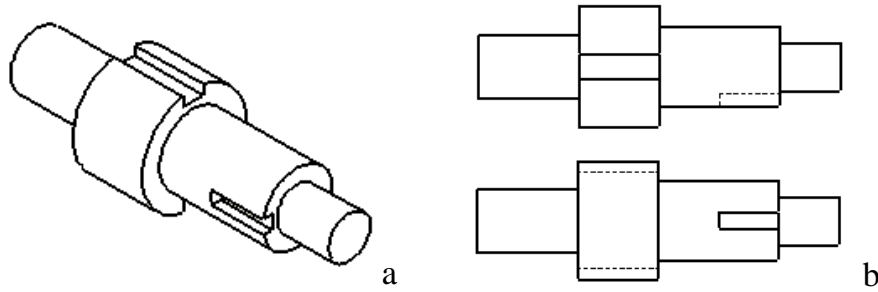

Fig. 22. Example 1 in axonometric view (a) and multiview (b)

Shafts usually contain slots such as the pair of opposed slots in the biggest section of our shaft. We deduce that a second part, a gear or hub, may slide into place there, as this displacement is allowed by the slots, and will then be constrained to rotate along with the shaft itself.

Our shaft contains a second, distinctive, slot, a keyway. We can deduce the presence of a corresponding feature to the second part, the gear or hub, but we would need to see this part too before deducing the keyway's function. In fact, the keyway, together with a similar keyway located in the gear or hub, and complemented by a key, produces an assembly in which the gear/hub is fixed to the shaft but may be separated if required.

Experimental Results: 88\% of those polled identified the object as an element of a transmission system (a shaft); 36\% specified its main function as power transmission; some people identified the object only as a connecting part (12\%).

Most opinions highlighted the importance of the existence of slots for accommodating keys (81\%) and a majority also highlighted the coaxial cylinders with different diameters on the part (56\%).

Most subjects deduced that these characteristics were related to the co-rotation of the part and another transmission component (75\%). Other explanations, while deducing the presence of one or more other components, described these as bearings or gears (17\%), as another shaft (8\%) or as an engine or motor $(8 \%)$.

One interesting response suggested that the part could be a component from a mechanism of a lock or a door knob.

Discussion: Our results show that this part is well known by engineers independent of their specialisation. Functional feature recognition as well as shape recognition is unquestionably essential for deducing the general purpose of the part, and also for deducing what other parts could be necessary in the assembly, and our subjects have reached general agreement about all of this.

\subsection{Example 2}

The example in Fig. 23 has an obvious large flat plate on its underside, and both the visible vertical hole and the semi-oblong hole through the plate (and the possible second holes if we assume that the part is symmetrical) suggests that this object not only rests on a surface but is fixed to it by screws or bolts. 
When we remove the base plate, we are left with two vertical dividers reinforced by four ribs each. Each of the dividers has a through hole, and the two holes are aligned, suggesting that something fits through them. The further fact that the holes are circular and unobstructed suggests that the second part is an axle and may rotate.

The presence of parts moving with respect to one another would mean that strength is important, so the ribs which are presumably there to strengthen the part support our interpretation: this part holds a rotating axle in place above a flat surface.

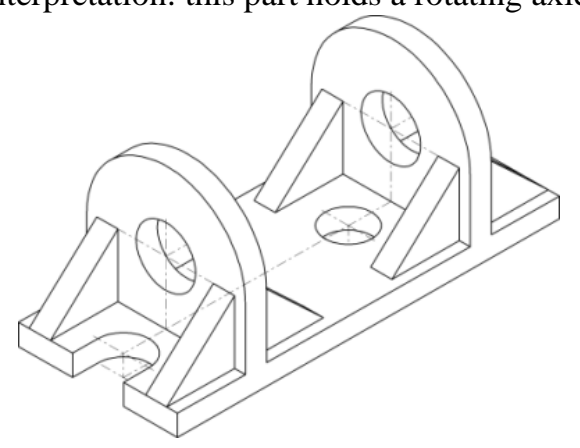

Fig. 23. Example 2

In fact, the part is a line shaft unit from an energy transfer kit which converts electrical energy via mechanical energy to potential energy. One end of the rotating shaft is driven by an electrical motor; the other end overhangs the base and winds up a weight, and stopping this weight from overbalancing the part is another reason why the part must be attached firmly to the flat surface.

Experimental Results: all subjects understood that the main function of the depicted object is a type of structural support (100\%). Some of them named the part as a base plate (38\%), a shaft/arbor support (38\%), a wire binding (6\%) or even a hinge $(6 \%)$.

$63 \%$ of subjects noted that the part could be fixed to a surface through the holes of its horizontal base.

In addition, 56\% described that coaxial holes (which seem to have the same diameter) in vertical dividers suggest a shaft or several cylindrical part(s) allowing a movement of rotation. Another 31\% disagreed, reasoning that the coaxial holes only supported or accommodated non-rotating parts (tools, objects, tubular elements, wires). The remaining 13\% thought that the disposition of the holes in the part could allow a rotational motion transmission between a horizontal and a vertical axis through, for example, bevel gears.

$19 \%$ of subjects indicated that the ribs imply that the part could withstand external forces, and their main function is to reinforce the structure.

Finally $13 \%$ suggested the possibility of using bearings in the coaxial holes in order to facilitate the rotational motion.

One interesting response suggested that the part could be part of a carpenter's workbench.

Discussion: Our results show that this part is quite well known by engineers independent of their specialisation, but there are two reasonable interpretations. All subjects agree about the support function of the part-as in example one, functional feature recognition contributes essential information for understanding the design intent of the part. While all of the subjects noted that the coaxial holes implied the presence of another part in the assembly, there was no general agreement about what that second part did: a majority said that it was a rotating shaft, but others disagreed. Finally, not many subjects described the function of the ribs in enhancing the support function of the part, even though this is an important clue to the design intent. In fact, the subjects seemed more concerned with finding alternative dispositions of the external shafts or objects.

\subsection{Example 3}

Fig. 23 depicts an assembly of three parts, of which it is clear that part 2 is the outer part. Again, the presence of a central axis and the basically cylindrical shape suggest that the assembly rotates.

Clearly, the slots in part 2 match the rails in part 1, so that is how the two parts must be assembled. This arrangement allows easy assembly by sliding the ring into the hub in the direction of the central axis, but prevents rotation about the axis. Thus the entire assembly rotates as one part.

We are left with the six bosses around the central hole of part 1 (they are clearly shown in the multiview drawing of part 1 in fig. 24b). There is little we can deduce from the geometry about the purpose of these bosses.
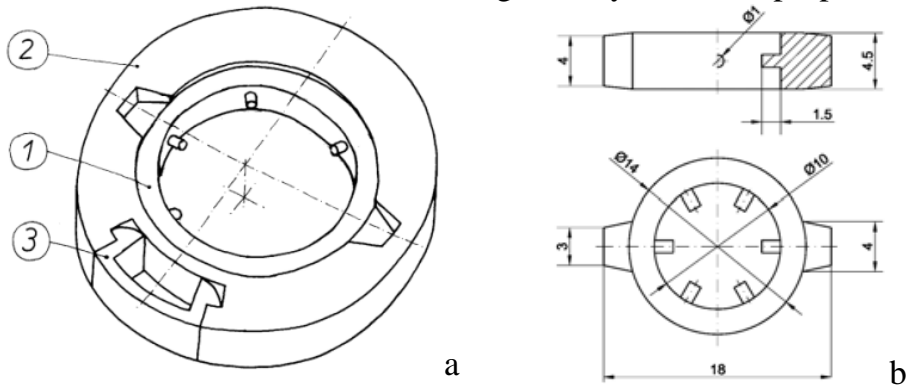
Fig. 24. Video-tape hub (a) and multiview of part 1 (b)

In fact, the figure shows one of the two spools of a video recorder. Tape is rolled up on the external surface of the drum (2). A peg (3) fixes the start of the tape to the drum external surface. The internal ring (1) fits in the rotating pulley of the video recorder. The pulley surface will contain indentations to match the six bosses of our inner ring, which cause the spool to rotate when the pulley rotates.

Experimental Results: In this example the subjects produced a greater variety of responses. The part was even named in several different ways, including drum (31\%), mounting bracket (“soporte de fijación”) (25\%), washer (19\%), bearing (13\%)... This shows that the function of this part has several interpretations.

Around $70 \%$ of subjects identified that part 1 is embedded in part 2 by their side plates. In addition, 56\% of subjects commented that the bosses of part 1 adjusted, positioned and centred an inner part; another 31\% thought that the main function of part 1 was to transmit rotary motion between the inner part and parts 2 and 3; while the remaining 13\% thought that it prevented rotational movement between the inner parts (2 and 3), acting as a bearing.

Other interesting comments were (a) that if the part were made from flexible material, it could eliminate vibrations to the inner part (6\%), and (b) that the bosses of part 1 reduced the friction with the inner part (6\%).

Discussion: Unlike the other two examples, in this case the part seems not to be well known by the subjects. Although most subjects recognised the individual features, there were widely differing deductions about the design intent of the whole part.

Our results suggest that designers first try to make sense of a whole part by a sort of template matching strategy, and use features to reinforce or reject this global matching. In those cases where their previous experience does not include anything similar, designers try to indirectly determine global functionality by using what they know about functionalities of individual features and determining which combination of candidate functionalities gives the best overall interpretation of the object.

\section{Conclusions and Future Work}

We have outlined how, by applying a reductionist approach, a drawing can be decomposed into its component form features. By analysing combinations of these form features, it should be possible to determine the design intent of the portrayed object. We have carried out simple experiments to determine (a) whether this approach corresponds to the way engineers habitually interpret drawings and (b)-whether it may be algorithmically replicated. The results of our experiments show that a) designers have a common knowledge base, a set of features which they can identify in 2D drawings, b), at least for the most common parts, they can guess their design intent, even if the parts are drawn alone and not contextualised, and c) they use the sort of reductionist approach we propose here as a fallback alternative, for parts they do not recognise.

We have identified and catalogued the most common form features found in engineering sketches. The catalogue is incomplete, and will remain so. Engineering designers are inventive, and will create new design features faster than we can keep up. Even so, detecting as many common form features as possible is still feasible and useful.

We have proposed geometric criteria (cues) by means of which these design features may be identified. Some of the criteria are subtle and subjective, such as the aspect ratio test in Section 3.9, where the threshold between what is and what is not a rib is undefined.

We have also proposed practical suggestions on how to map some common shape features perceived in sketches into their corresponding design features.

In future work, we shall introduce and implement algorithms for identifying those form features for which none yet exist, and we shall formalise and implement a reasoning process similar to that used in Section 4 to deduce design intent from the various form features present in a part.

Acknowledgments This work was partially funded by financial support from the Ramon y Cajal Scholarship Programme and by the "Pla de Promoció de la Investigació de la Universitat Jaume I", project P1 1B2010-01.

\section{References.}

1. Babic B, Nesic N, Miljkovic Z (2008) A review of automated feature recognition with rule-based pattern recognition. Computers in Industry, 59(4): 321-337

2. Bartolo A, Camilleri KP, Fabri SG, Borg JC, Farrugia PJ (2007) Scribbles to Vectors: Preparation of Scribble Drawings for CAD Interpretation. Proceedings of the Eurographics Workshop on Sketch-Based Interfaces and Modeling 2007, 123-130

3. Bonnici A, Camilleri KP (2009) Scribble Vectorization Using Concentric Sampling Circles. 3rd Int. Conf. on Advanced Engineering Computing and Applications in Sciences (ADVCOMP 2009), 89-94

4. Booker P J (1979) A History of Engineering Drawing. Northgate Publishing Co. London

5. Company P, Contero M, Varley PAC, Aleixos N, Naya F (2009) Computer-Aided Sketching as a Tool to Promote Innovation in the New Product Development Process. Computers in Industry 60 (8), 592-603

6. Company P, Varley PAC (2010) A Method for Reconstructing Sketched Polyhedral Shapes with Rounds and Fillets. Smart Graphics 2010 Proc. (LNCS 6133), 152-155

7. Cooper M (2008) Line Drawing Interpretation, Springer 
8. CustomerPartNet. http://www.custompartnet.com/lessons/. Accessed 15 November 2011

9. Ferguson ES (1992) Engineering and the Mind's Eye, MIT Press

10. Han JH (1996) Survey of Feature Research. Technical Report IRIS-96-346, University of Southern California Institute for Robotics and Intelligent Systems

11. Han JH (1997) On Multiple Interpretations. In ed. C.Hoffmann and W.Bronsvort, Proceedings: Fourth Symposium on Solid Modeling and Applications, 311-321, ACM Press

12. Han JH, Requicha AAG (1996) Geometric Reasoning for Feature Recognition. Technical report IRIS-95-343. Extended version of "Hint Generation and Completion for Feature Recognition” $29^{\text {th }}$ International Symposium on Automotive Technology and Automation (ISATA), Florence, Italy, pp 8996

13. Han JH, Pratt M, Regli W (2000) Manufacturing Feature Recognition from Solid Models: A Status Report, IEEE Trans. on Robotics and Automation, 16(6), 782-796

14. Hilaire X, Tombre K (2006) Robust and accurate vectorization of line drawings. IEEE Transactions on Pattern Analysis and Machine Intelligence, 28(6), 890 - 904

15. Piquer A, Company P, Martin RR (2003) Skewed mirror symmetry in the 3D reconstruction of polyhedral models. Journal of WSCG 11(3), 504-511.

16. Shahin TMM (2008) Feature-Based Design - An Overview. Computer-Aided Design and Applications 5(5), pp 639-653

17. Sugihara K(1986) Machine Interpretation of Line Drawing. MIT Press

18. Thomson WB, Owen JC, De St Germain HJ, Stark Jr SR, Henderson TC (1999) Feature-based Reverse Engineering of Mechanical Parts. IEEE Transactions on Robotics and Automation 15 (1), pp. 57-66

19. Tombre K (1998) Analysis of engineering drawings. GREC’97 (LNCS 1389), 257-264

20. Vandenbrande JH, Requicha AAG (1993) Spatial Reasoning for the Automatic Recognition of Machinable Features in Solid Models, IEEE Transactions on Pattern Analysis and Machine Intelligence, 15(12), 1269-1285

21. Varley PAC (2003) Automatic Creation of Boundary-Representation Models from Single Line Drawings, PhD Thesis, University of Wales

22. Yaner PW, Goel AK (2006) From Form to Function: From SBF to DSSBF. In Proc. Second International Conference on Design Computing and Cognition, Eindhoven, Netherlands

23. Yaner PW, Goel AK (2008) Analogical recognition of shape and structure in design drawings Artificial Intelligence for Engineering Design, Analysis and Manufacturing: AIEDAM 22 (2) , pp. 117-128

24. Zhang X, Song J, Dai G, Lyu M R (2006) Extraction of line segments and circular arcs from freehand strokes based on segmental homogeneity features. IEEE Trans. Systems, Man, and Cybernetics, 36 (2), 300 - 311 